\title{
Beacon: bentonite mechanical evolution
}

\author{
Patrik Sellin ${ }^{1, *}$, Mary Westermark ${ }^{1}$, Olivier Leupin ${ }^{2}$, Simon Norris $^{3}$, Antonio Gens ${ }^{4}$, Klaus Wieczorek ${ }^{5}$, \\ Jean Talandier ${ }^{6}$, and Johan Swahn ${ }^{7}$ \\ ${ }^{1}$ Svensk Kärnbränslehantering AB (SKB), Evenemangsgatan 13, Box 3091, 16903 Solna, Sweden \\ ${ }^{2}$ Nagra, Hardstrasse 73, Postfach 280, 5430 Wettingen, Switzerland \\ ${ }^{3}$ Radioactive Waste Management, Building 587, Curie Avenue, Harwell Oxford, Didcot, Oxfordshire OX11 0RH, UK \\ ${ }^{4}$ Department of Civil and Environmental Engineering, Universitat Politècnica de Catalunya (UPC) - BarcelonaTech, \\ Jordi Girona 1-3, Edifici D-2, 08034 Barcelona, Spain \\ ${ }^{5}$ Gesellschaft für Anlagen- und Reaktorsicherheit (GRS) gGmbH, Abteilung Standortauswahl, Bereich Endlagerung, \\ Theodor-Heuss-Str. 4, 38122 Braunschweig, Germany \\ ${ }^{6}$ Andra, 1/7, rue Jean Monnet, Parc de la Croix-Blanche, 92298 Châtenay-Malabry Cedex, France \\ 7 Miljöorganisationernas kärnavfallsgranskning (MKG), Första Långgatan 18, 41328 Göteborg, Sweden
}

Received: 12 March 2019 / Accepted: 16 September 2019

\begin{abstract}
The aim of Beacon is to develop the understanding of fundamental processes that lead to material homogenisation, as well as to improve capabilities for numerical modelling. In earlier assessments of bentonite EBS, the mechanical interaction between the installed bentonite components has been neglected and an "ideal" final state has generally been assumed. Key features of the project are (1) re-evaluation of the available knowledge to extract the crucial data to compile the qualitative and quantitative data and to enhance the conceptual understanding. (2) Enhanced, robust and practical numerical tools based on a good scientific understanding, which have the expected predictive capabilities regarding the evolution of engineered barriers and seals. (3) A developed database with experimental data needed by the quantitative models. (4) Verified calculation tools based on experimental results in different scales. The Beacon project is required for the panEuropean objectives at building confidence amongst regulators and stakeholders regarding the performance of the engineered barriers in a geological repository.
\end{abstract}

\section{Introduction}

The key objective of Beacon [1] is to refine and verify the tools needed for the assessment of the mechanical evolution of installed bentonite components and the eventual performance of the barrier. The aim is to demonstrate that the performance of present designs for buffers, backfills, seals and plugs is appropriate. In addition, for repository designs particularly in crystalline host rock, the results may also be used for the evaluation of effects of mass loss from a bentonite barrier in long term perspective.

The driver for this project is repository safety, and the demands of waste management organizations to verify that the material selection and initial state design fulfil the longterm performance expectations. For this project, the initial state refers to the period of installation of the barrier, while

\footnotetext{
* e-mail: patrik.sellin@skb.se
}

long-term performance refers to the period for barrier saturation and evolution of the hydro-mechanical state, which could range from 10 to 1000s of years. In current and future applications for repositories, the regulators will expect the applicants to have a sufficient predictive capability of the barrier evolution from the installed to the final state.

Beacon is focused on the direct application to real assessment cases in actual repository systems. A few cases from relevant repository systems have therefore been selected as test examples. The systems intended to be evaluated in Beacon include three cases: (1) a tunnel plug based on the ANDRA design, (2) a disposal cell from the Nagra concept, (3) the KBS-3 deposition tunnel backfill. These are representative of the primary areas of uncertainty in density homogeneity. These examples cover a broad range of issues and the results should be applicable to other concepts and systems as well. The cases are illustrated in Figures 1-3. 


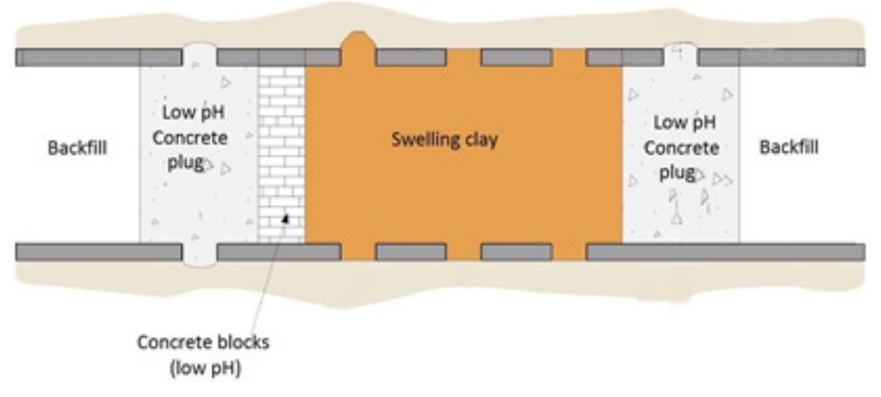

Fig. 1. Tunnel plug in the ANDRA concept.

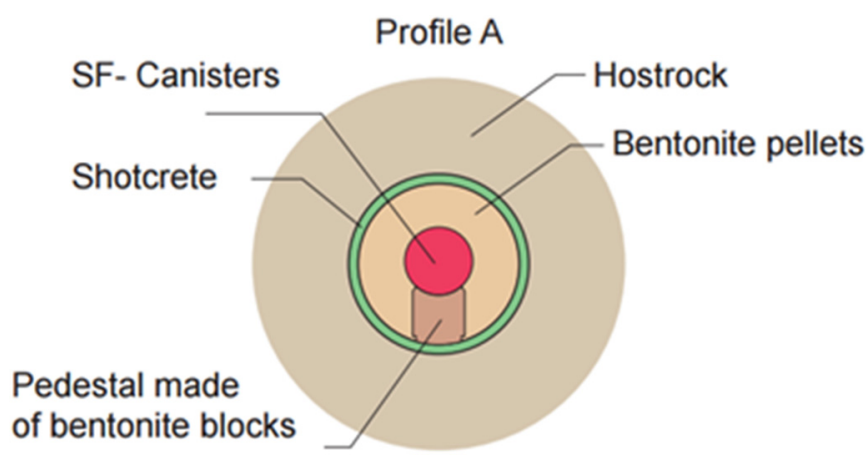

Fig. 2. Disposal cell in the Nagra concept.

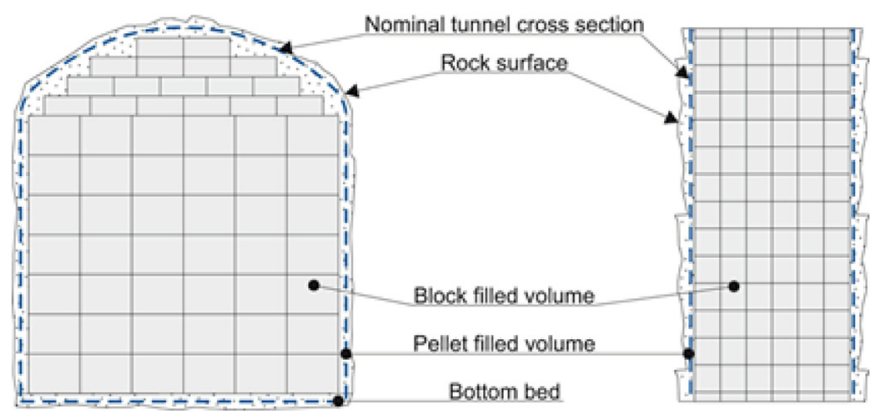

Fig. 3. KBS-3 Tunnel backfill.

Beacon builds upon experience by waste management organizations from different countries and technology providers over the past $30+$ years. Great gains have been made in understanding individual bentonite components through experimental and modelling work. Yet shortcomings in the state-of-the-art knowledge exist which still inhibit confidence toward repository operation.

The sealing ability is crucial for bentonite barriers in all geological repository concepts. Sealing is achieved by the combination of a high swelling pressure and a low hydraulic conductivity. The swelling pressure will impact the other barriers in the repository as well. The mechanical properties of the installed EBS, that will consist of a combination of blocks, pellets and voids, will be completely different from the final state after full saturation. It is therefore crucial to consider:

- the mechanical evolution during the saturation phase;

- the final state at equilibrium.

A firm understanding of the mechanical evolution is necessary to ensure that a given design will meet the expected performance targets.

The scientific-technical work in Beacon is structured in five work packages (WP1-5), dissemination and training is handled in two work packages (WP6-7) while coordination and project management is covered in one single work package (WP8). The interconnections between the work packages are illustrated in Figure 4.

WP1 is the main driver for the entire project. The waste management programs involved are all represented in WP1, through the implementer or equivalent organization. The objective of $\mathbf{W P 1}$ is to define the important issues concerning the mechanical properties of bentonite and to define how these should be treated.

In WP2 the existing knowledge base is treated. The key objective of WP2 is the sharing of knowledge and experience. The partners have distributes information and results from earlier assessments, design considerations, experiments and modelling tasks.

A strong driver for a joint project is the current limitations in the predictive capability in the numerical models. The issue of homogenisation and swelling is challenging both from a conceptual and a numerical point of view. The purpose of WP3 is to identify and resolve the shortcomings of current models.

Although there is a substantial experimental database available for the project, it is necessary to perform additional experiments to support the model development in WP3 and the model testing in WP5. The experimental work is coordinated in WP4. WP4 consists of experienced experimental groups, which have the flexibility to adapt the experimental work to support the needs of WP3 and WP5.

The core component of Beacon is WP5. The main effort is performed in this work package. The overall objective of WP5 is to simulate the assessment cases defined by WP1. In order to do this, the available models have to be tested first on results from laboratory experiments and later on results from large scale field tests to gain confidence in their predictive capability. The next step is to actually test the predictive capability, by means of "blind" predictions of experimental results. Finally, the models will be used to evaluate the assessment cases.

\section{WP1 definition of assessment case/ application to the assessment cases}

The needs in safety assessments regarding the evaluation of heterogonous backfill properties are addressed, in particular to what extent heterogonous material property distributions will comply with performance targets are addressed in WP1. The outcome of this work package is expected to be a (hydro)-mechanical assessment of the selected assessment case, considering a range of 


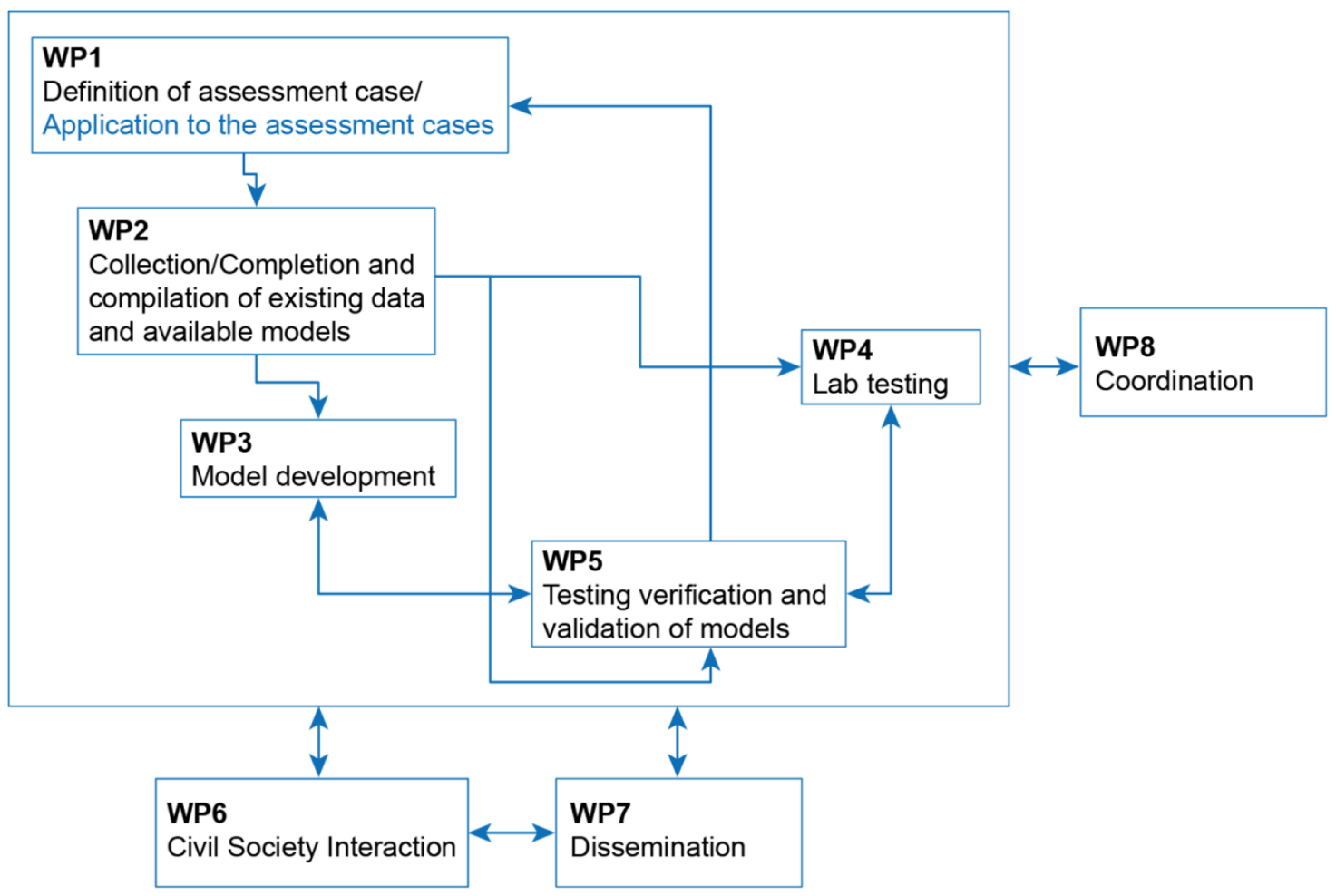

Fig. 4. Interconnections between the work packages in Beacon.

uncertainties in the boundary conditions that would eventually result in a set of requirements under consideration of the host rock and the repository design.

The first deliverable was a report compiled with the answers to a questionnaire that was distributed to the different WMOs or their representatives. The questionnaire aimed at reflecting the state-of-the-art regarding the treatment of heterogeneous bentonite density distribution and properties in the safety case. The questionnaire consisted of three different parts: (1) application of bentonite in the specific design; (2) the required performance of bentonite; (3) detailed characterization of the required properties of the bentonite.

The conclusions from the answers to the questionnaire were that occurrence of heterogeneity in the repositories could impact the safety functions of bentonite components. Therefore, it needs to be determined to what extent this could affect the safety case of the repositories. Heterogeneity can occur in the initial material, through the emplacement or the re-saturation phase as well as on the long term after re-saturation of all repository components. The heterogeneities in the initial state, i.e. after installation of the EBS, are mainly due to density differences. Inhomogeneous saturation and swelling of bentonite could cause irreversible damage. The role of uncertainties related to these bentonite heterogeneities is addressed in most repository concepts using a deterministic approach, defined with a preferred density value. There are several natural properties of bentonite that may impact the degree of homogenization. Most waste management organizations consider water content, original exchangeable cations, bulk density, swelling pressure and hydraulic conductivity as relevant natural properties for the bentonite regarding heterogeneity, while organic carbon or thermal conductivity seem to be incidental to the homogenization process.

All participating waste management organizations agree that the most valuable output from Beacon would be material models that are accurate enough to be used as a tool for design and engineering purposes, i.e. to assess the behaviour and performance of the bentonite-based EBS both on the short- and long-term under variable design and environmental conditions. It is expected that, if preparation of the sealing material (e.g. pellets) and emplacement method are performed properly, heterogeneity will not be problematic for safety cases and the buffer material can be represented in the safety assessment by a well-chosen homogeneous material.

\section{WP2 collection and compilation of existing data and available models}

The most important outcome of WP2 so far has been a report that documents the information that has been made available to the Beacon project by the project partners and associated organisations. The information relates to experiments at a number of different scales as well as modelling studies that have been undertaken. The report utilizes information provided by Beacon partners regarding experiments that have been carried out in earlier projects, with the purpose to build a database of experiments which can be used during the Beacon project and even beyond. 
The report documents the available data but does have the purpose to propose any experiments for consideration within the Beacon project. This objective will be handled by the Work Package leaders, based on the information contained in the report. The report provides clear referencing to the underlying reports which contain further information, to facilitate the process of selecting datasets for modelling within Beacon. The process for collecting and compiling information into the database involved:

- creating a designated data form to collect appropriate information;

- requesting that Beacon partners and associated organisations fill out the form for any studies that could be relevant to Beacon;

- collation of the completed data forms into a database;

- arranging a workshop for the discussion of the database and definition of additional fields that would aid selection of experiments for modelling cases within Beacon;

- request for additional information to complete the new fields in database;

- completion of the database.

The data forms entered into the database have been divided in three categories: laboratory experiments, mock-up experiments and in situ experiments. The laboratory experiments include both experiments with the purpose to measure material properties, as well as experiments that simulate repository conditions in a smaller scale. There are many experiments listed in the database, at a range of scales (from bench top laboratory experiments to full scale field experiments), a small number of these were designed specifically for studies of bentonite homogenisation, but the majority were originally designed for other purposes. Nevertheless, all these experiments provide a valuable source of information on the mechanical properties of bentonite and the mechanical evolution of a bentonite barrier within a repository. The database contains information on the type of bentonite considered in the tests, the boundary conditions and initial and final heterogeneities within the experiments, but also the range of measurements taken in the experiment. This will give Beacon partners and especially Work Package leaders the opportunity to interrogate the database and find experiments of interest for consideration as case studies within the Beacon project. Furthermore, knowledge about experiments that have been undertaken previously will guide decisions on new experiments to be undertaken.

\section{WP 3 model development}

Work Package 3 plays a central role in the structure of the project as it is devoted to the development of the constitutive models for describing the hydro-mechanical behaviour of the bentonite in an appropriate manner. It is recognized that current models face limitations in their predictive capabilities and significant advances are required. The models must prove their predictive capabilities, reliability and robustness and they should preferably be grounded on a good understanding of the phenomena involved. To this end, they will be validated using several

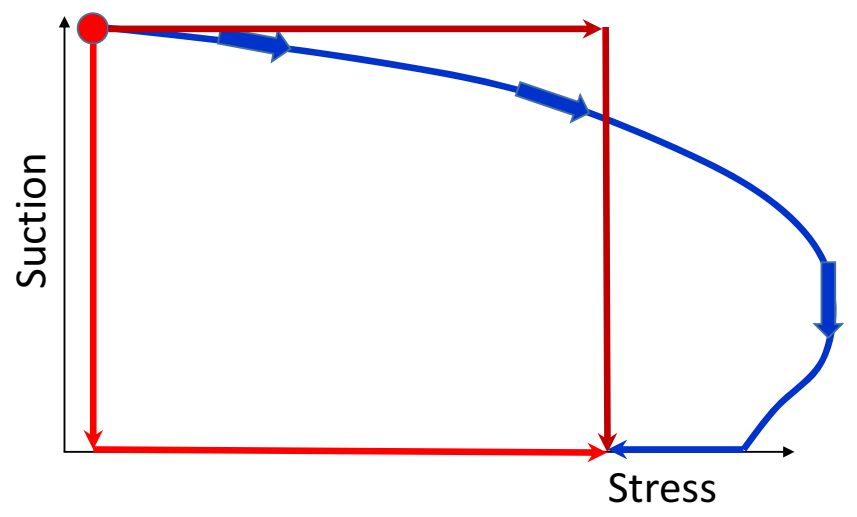

Fig. 5. Suggested suction-stress paths to check stress path dependency from an unsaturated to a saturated state. Stress may be vertical total stress (in oedometer tests) or mean total stress (in isotropic loading tests).

laboratory and field-scale tests. Ideally, those constitutive models should consider the following cases:

- saturated and unsaturated materials;

- compacted bentonite (Blocks) and granular bentonite (e.g. pellet-based);

- isothermal and non-isothermal conditions,

although it is recognised that not all models will necessarily have this comprehensive level of generality.

To facilitate a more common assessment of the models, the teams were asked explicitly what the model capabilities were concerning a number of features of behaviour:

- dependence of swelling strain on applied stress and on dry density;

- irreversibility of strains in wetting/drying cycles;

- behaviour during swelling stress test. Dependence of swelling pressure on dry density;

- stress path dependence from an unsaturated to a saturated state (Fig. 5);

- stress path dependence from a saturated to an unsaturated state;

- dependence of strains developed in a temperature cycle (increase/decrease) on OCR (Overconsolidation Ratio) (or stress).

The first five items correspond to an isothermal formulation whereas the sixth one requires the incorporation of temperature effects. The features selected for this purpose are those that are deemed, in principle, most relevant to explain the evolution of engineered barriers and seals during the transient phase.

The model capabilities at the beginning of the project are available as deliverable 3.1 .

\section{WP4 lab testing}

The objectives of the Beacon experimental studies are to provide input data and parameters for development and validation of models and to reduce uncertainties about conditions and phenomena influencing bentonite homogenisation. Both the homogenisation of an initially inhomogeneous bentonite system and the persistence or 

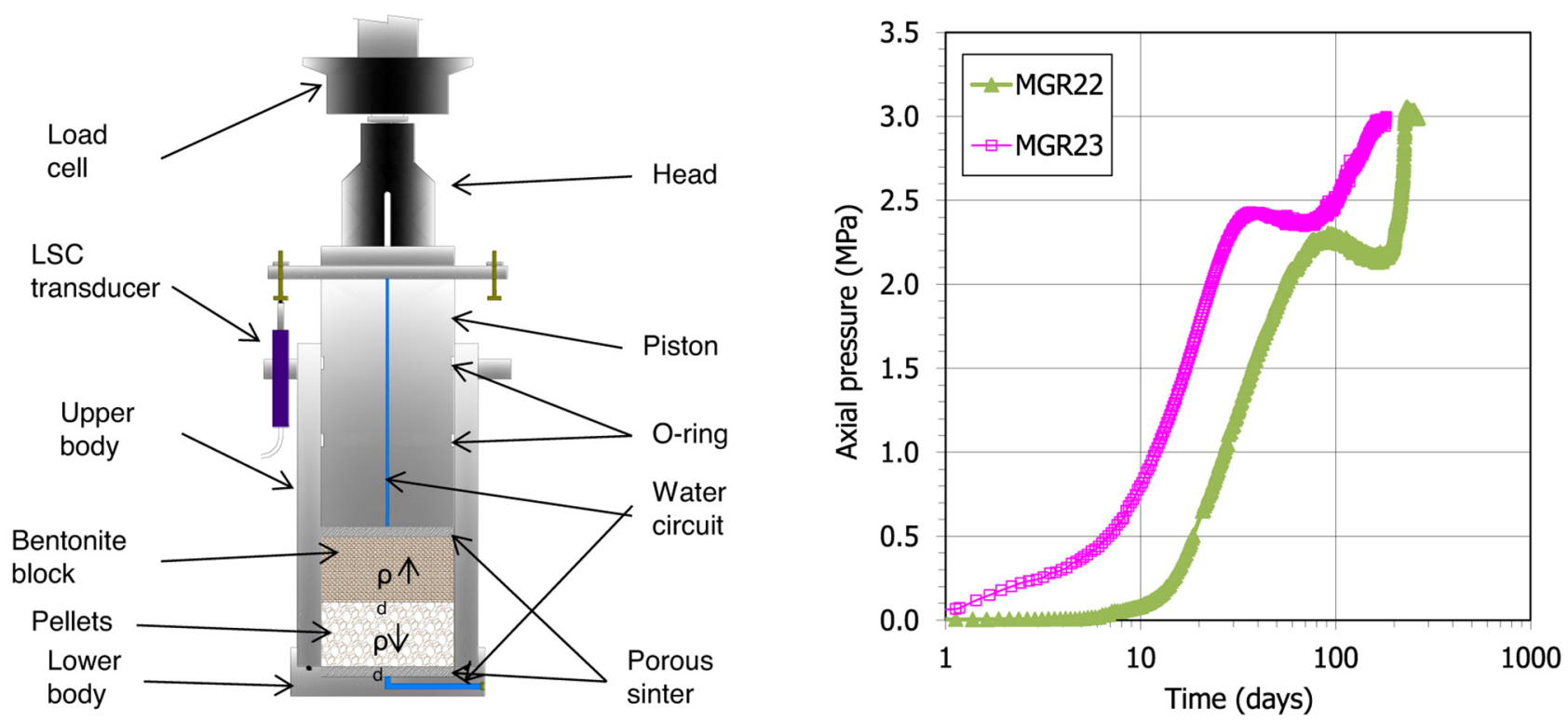

Fig. 6. Isochoric cell for hydration tests on bentonite block/pellets and swelling pressure evolution in two tests.

development of inhomogeneities in the bentonite systems under various mechanical and hydraulic conditions are investigated. Eight experiment teams perform tests involving different bentonite materials and hydraulic and mechanical boundary conditions. As an example, CIEMAT's hydration tests in large-scale isochoric cells are presented here.

The work focuses on the conceptual understanding of the evolution of bentonite fabric and microstructure upon hydration and the factors affecting them. To accomplish this, hydration tests are being performed in isochoric cells (Fig. 6) to analyse the fabric and microstructure evolution of initially inhomogeneous FEBEX bentonite. In the tests half of the sample was composed of bentonite pellets (initial dry density $1.3 \mathrm{~g} / \mathrm{cm}^{3}$ ) and the other half of a bentonite block (initial dry density $1.6 \mathrm{~g} / \mathrm{cm}^{3}$ ). The two samples were prepared in the same way, but one of them was hydrated under a constant flow rate of $0.05 \mathrm{~cm}^{3} / \mathrm{h}$ (MGR22) and the other one under a constant injection pressure of $14 \mathrm{kPa}$ (MGR23). The first boundary condition tried to simulate a host rock with limited water availability, whereas the other one simulated a repository with plenty of water in which the water intake is controlled by the bentonite permeability. The axial pressure development in the two tests is shown in Figure 6. There was a significant difference in swelling development kinetics, but although it took much longer to reach an equilibrium value for the test performed under low water inflow (MGR22), the final swelling pressure value, once the samples were saturated, was the same in the two cases, about $3 \mathrm{MPa}$, which is the expected value for a granular FEBEX sample compacted to the average dry density of the block/pellets set $\left(1.42 \mathrm{~g} / \mathrm{cm}^{3}\right)$.

Once the sample from test MGR22 was dismantled, its physical state was checked by determining the water content, dry density and pore size distribution at different positions. The final appearance of the clay was homogeneous, with no discernible pellets. There was a slight decrease of water content from the hydration surface to the top of the sample (from $35 \%$ to $30 \%$ ), while the dry density increased in this sense (from 1.39 to $1.46 \mathrm{~g} / \mathrm{cm}^{3}$ ). The average dry density of the bentonite block considerably decreased as a consequence of saturation and that of the pellets part increased. The degree of saturation was close to $100 \%$ in every position. The pore size distribution significantly changed with respect to the original samples: the macroporosity of the pellets decreased, whereas the microporosity of both the pellets and of the block increased.

\section{WP5 testing verification and validation of models}

One of the objectives of Beacon project is to improve models to simulate bentonite component evolution along the repository life. In this context, a specific work package is dedicated to verification and validation of models.

Based on an inventory performed within the project identifying experiments in link with mechanical evolution of repository bentonite barrier materials, a selection of test cases were made. The test cases are based on experiments performed at several scales: from lab tests $(\mathrm{cm})$ to field tests (scale 1) which are relevant in regards of the Beacon objectives.

Partners involved in WP5 make simulations on the chosen test cases and produce specified results. The objective of those simulations is not only to reproduce the experimental results which most of the time could be done by identifying some relevant parameters but also to detect where the difficulties in terms of modelling are. The aim is to be able to improve our capacities of prediction of 
long-term behaviour for bentonite based components. Comparisons of results and analyses of the differences will lead to an improvement of the physical and numerical models creating a strong link with WP3 (model development), and should give a feedback to the experimentalists involved in the dedicated work package (WP4).

The strategy was to select tests at laboratory scale where homogenization processes have been highlighted, which will constitute elementary bricks to tackle bentonite evolution modelling at a larger scale. The selected tests are: - swelling pressure tests for compacted plugs with free volume available TEST B1.7 from Clay Technology AB; - swelling pressure tests for pellets mixture TEST B1.16 from CEA, Andra;

- swelling pressure tests for block and pellets structure TEST B1.6 from Posiva.

The first test for modelling during this exercise was a MX-80 bentonite with an initial dry density of $1655 \mathrm{~kg} / \mathrm{m}_{3}$. The test had two successive phases (Fig. 7). The first phase was a classical swelling pressure test at constant volume. At stable swelling pressure, the upper piston was released and fixed at a higher position creating a certain volume for expansion. A second stage of swelling started when the expanding bentonite had the new volume.

At equilibrium, i.e. when no or negligibly small changes are seen in the recorded swelling pressure, the sample is dismantled and sliced axially to determine the water content and distribution of density in the direction of expansion.

Several partners modelled the test obtaining results in good agreement with the measurements at the end of the first stage for the axial swelling pressure (Fig. 8).
The exercise shows the difficulties of the models to represent the transient phase that is constituted mainly by water saturation and development of swelling pressure.

At the top of the sample, dry density and water content at the end of the test are higher due to the introduced void during the test. The water content obtained at the end of the test when the sample was dismantled is compared to the numerical results (Fig. 9) showing that the general trend for water content is well reproduced. Models seem able to reproduce the final heterogeneity of properties observed at the end of the test.

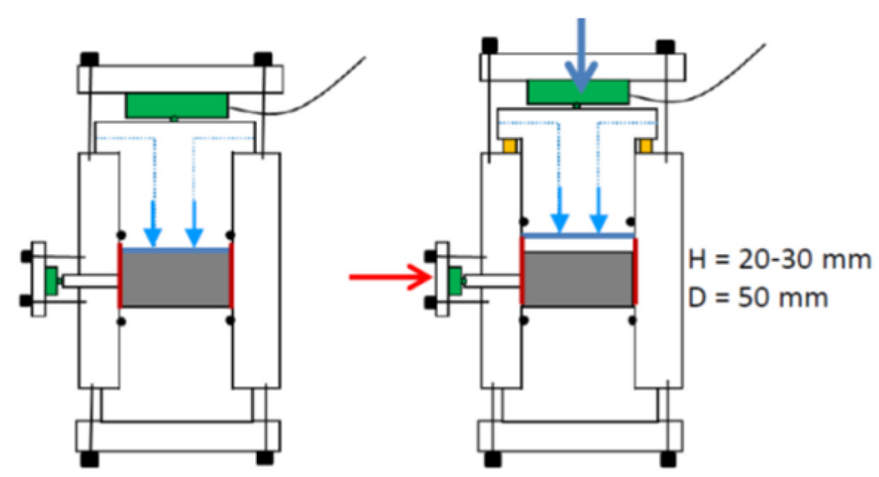

Fig. 7. Schematic of the axial swelling tests. The red lines show the lubricated walls and the blue lines indicate filters and water supply. The radial pressure transducer is located $10 \mathrm{~mm}$ above the bottom of the specimen.

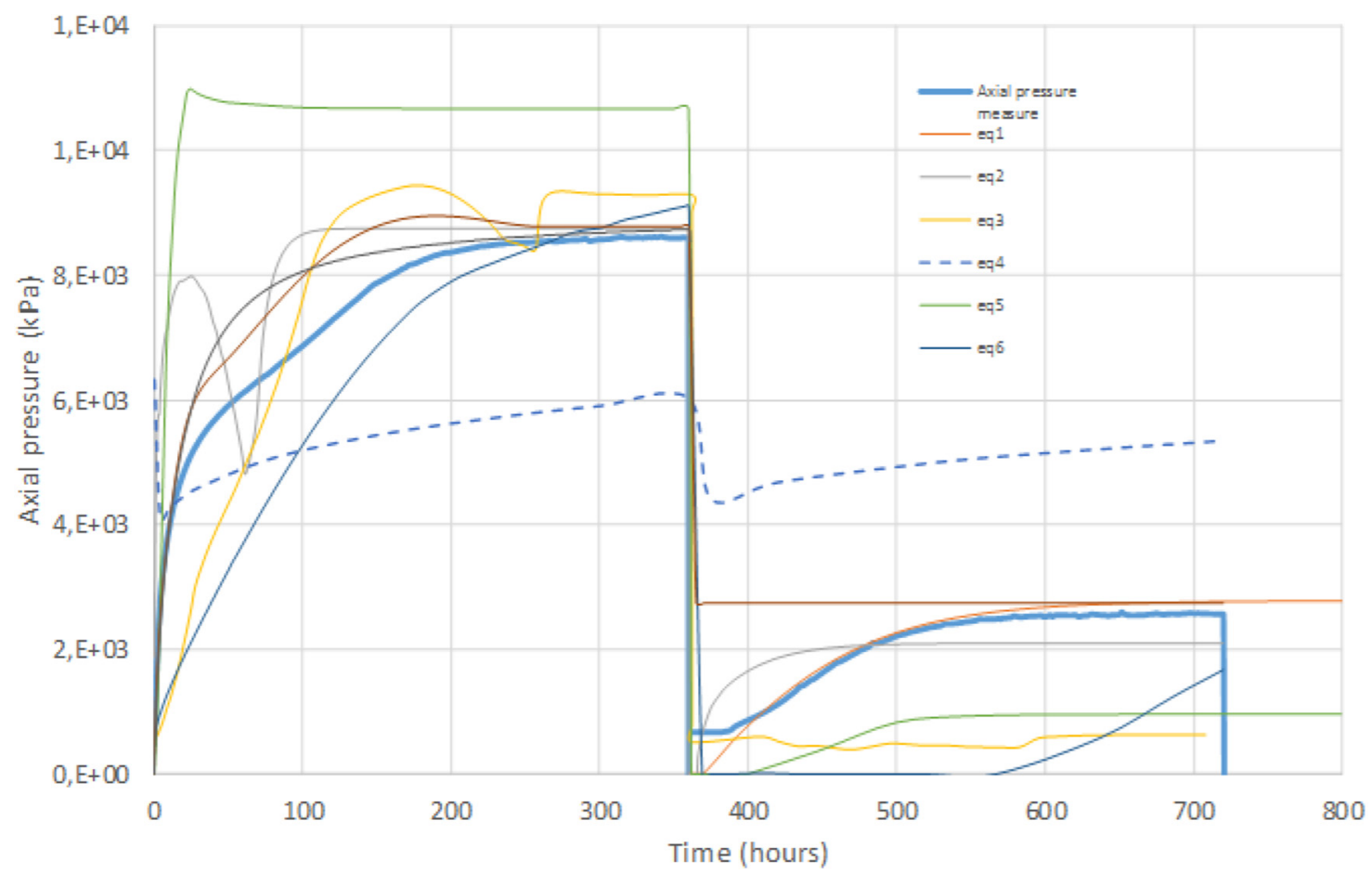

Fig. 8. Axial pressure evolution - comparison between the experiment and the models. 


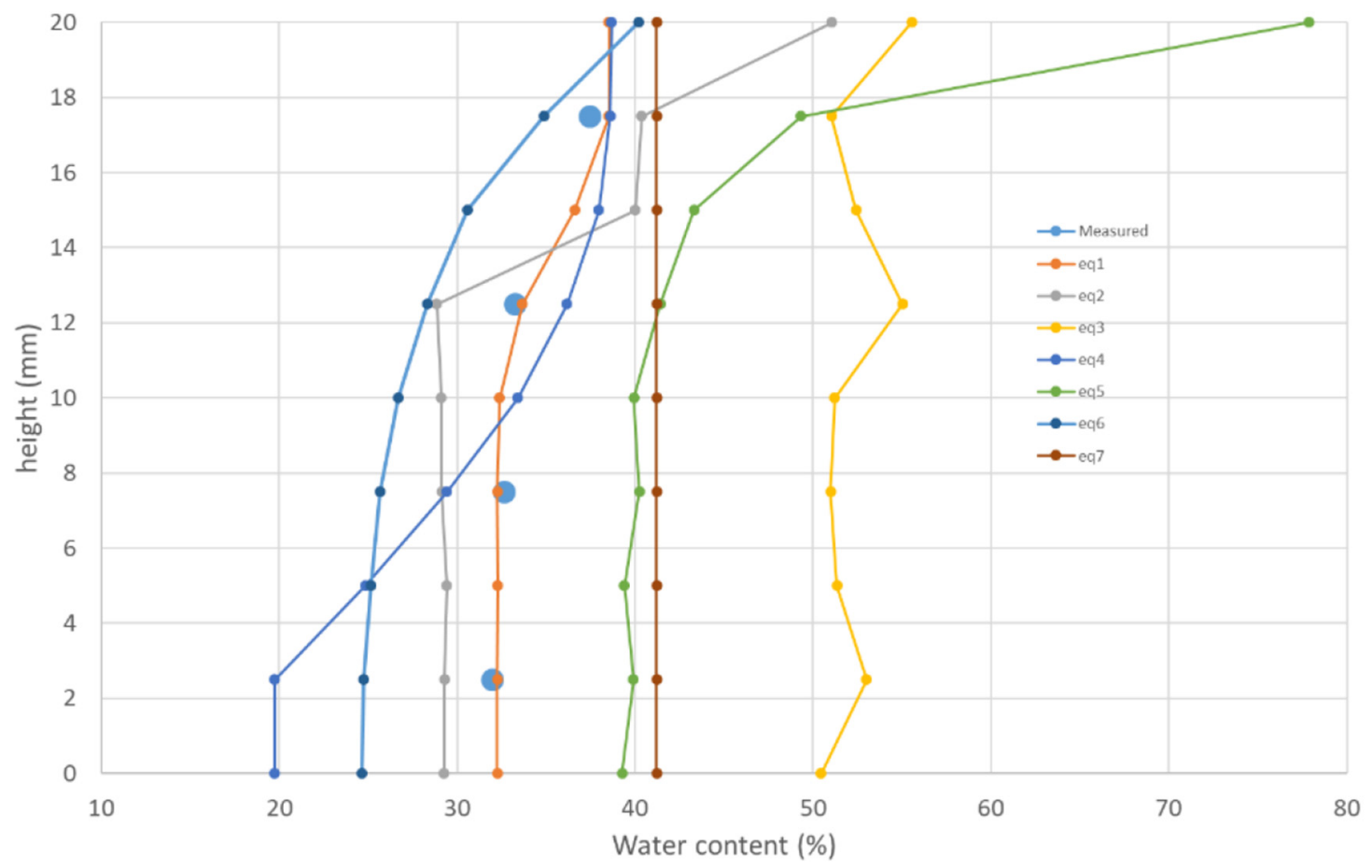

Fig. 9. Water content at the end of the test - comparison between the experiment and the models.

The first analysis was very useful to identify some specific points that need to be investigated and on what the model evolutions should be focused. The second test in the list above, performed on pellets, highlighted the same kind of conclusions. The hydration phase is always difficult to capture with the models.

The next task will be to model large scale field tests to show the capacity of the models to reproduce in situ experiments. Three experiments have been selected: EB Engineered Barrier Emplacement Experiment (Mont Terri), FEBEX - Full-scale Engineered Barrier Experiment in Crystalline Host Rock (Grimsel), CRT - Canister

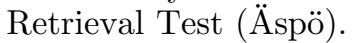

\section{Civil society interaction, dissemination and coordination}

The overall aim of WP6 is involve civil into the research performed in the Beacon project. Representatives from civil society are taking part in project and work package meetings to gain an inside information about the results. The aim of the work package is to facilitate the interpretation of scientific results and other output from WP1-5 to the interested public and enable for civil society local and national representatives to interpret, discuss and elaborate on the research results and other information generated by the project. This will prepare civil society for active participation in future situations where there are consultation processes as a part repository inplementation.

The Beacon project has arranged one specific training course during the project, within WP7. The course took place at the Universitat Politècnica de Catalunya (Spain).The course aimed to give an overview of the current approaches and capabilities concerning the constitutive and numerical modelling of the hydro-mechanical behaviour of bentonite and other swelling clays. The context of the course was the field of nuclear waste management; however, the concepts and methods that were presented have a much wider scope of application. The topics of the course were:

- the fundamental science behind the mechanical and hydraulic properties of bentonite;

- current constitutive modelling approaches;

- numerical modelling and examples of application;

- the issues around the mechanical evolution of bentonite in nuclear waste management;

- hands-on training with a computer code.

The course had 36 participants and addressed the nuclear waste management community as well as to students in areas of soil and material science and civil, environmental and mechanical engineering.

\section{Conclusion}

BEACON is now about half way into the project. So far the work has been both focused and according to plan. The 
State of Art reports from WP2 and WP3 are important summaries of the knowledge that never has been collected in a similar manner before. They will both be an important foundation for current and future mechanical assessments of bentonite. The benchmark tests in WP5 have also been very successful: they have pointed out that there are real challenges, but also have shown that there are model concepts available that should be able to meet those challenges.
The BEACON project receives funding from the Euratom research and training programme 2014-2018 under grant agreement No 745942 .

\section{Reference}

1. http://www.beacon-h2020.eu

Cite this article as: Patrik Sellin, Mary Westermark, Olivier Leupin, Simon Norris, Antonio Gens, Klaus Wieczorek, Jean Talandier, Johan Swahn, Beacon: bentonite mechanical evolution, EPJ Nuclear Sci. Technol. 6, 23 (2020) 